\title{
Docetaxel causes lymphatic endothelial cell apoptosis and impairs lymphatic function and gene expression in vitro
}

\author{
Wong AM, Baik JE, Park H, Li C, Shin JY, Kataru RP and Mehrara BJ* \\ Division of Plastic and Reconstructive Surgery, Department of Surgery, Memorial Sloan Kettering, Cancer Center, New York, New York 10065, USA
}

\begin{abstract}
Introduction: Recent studies have shown that taxanes — chemotherapeutic agents commonly used for breast cancer treatment - may increase the risk of lymphedema development in breast cancer survivors. The purpose of this study was to analyze the effects of docetaxel on lymphatic endothelial cells (LEC) and define the cellular mechanisms that may account for this clinical relationship.

Methods: Human dermal LECs were cultured in vitro with varying concentrations of docetaxel and LEC viability, proliferation, migration, tubule formation and lymphatic gene expression were analyzed.

Results: Docetaxel, at a concentration of $100 \mu \mathrm{M}$, was cytotoxic to LECs resulting in impaired proliferation. At the lower concentrations ( 1 and $10 \mu \mathrm{M})$, docetaxel impaired LEC function by decreasing LEC migration and tubule formation. The expression of lymphatic markers podoplanin, LYVE1 and FLT4, but not PROX-1 were down-regulated in LECs following high concentration of docetaxel treatment $(100 \mu \mathrm{M})$.

Conclusion: High concentration of docetaxel induces LEC death and impair LEC proliferation and lymphatic gene expression. In contrast, low concentration of docetaxel significantly impairs LEC migration and tubule formation. These adverse effects of docetaxel may therefore provide a cellular mechanism underlying the clinical observation that taxane therapy increases the risk of lymphedema development in cancer patients.
\end{abstract}

\section{Introduction}

Lymphedema is a chronic disease characterized by tissue swelling resulting from lymphatic fluid accumulation and fibroadipose tissue deposition [1]. In the United States, the most common cause of lymphedema is breast cancer treatment and the disease develop in $20-40 \%$ of women who undergo axillary lymph node dissection $[1,2]$. This surgical procedure interrupts lymphatic capillaries resulting in progressive accumulation of interstitial fluid due to lymphatic vessel leakiness, impaired lymphatic pumping causing tissue fibrosis, and adipose deposition.

Patients with lymphedema complain of limb swelling, pain, heaviness, decreased arm function, skin changes and thickening, scar tissue formation, and recurrent infections. As a result, lymphedema causes severe impairment in quality of life $[1,3]$. However, despite the fact that lymphedema is common and a major source of morbidity, treatments for this disease remain palliative and primarily rely on massage, skin care, and compression bandaging [3]. These therapies are effective in some patients but are not curative and are expensive both in terms of time and treatment costs; as a result, palliative treatments are often associated with poor compliance and disease progression. Thus, identification of risk factors that may contribute to lymphedema development as well as new treatment options for this disease is an important biomedical goal.

Obesity and nodal basin radiation are known to increase the risk of developing lymphedema following axillary lymph node dissection [47]. Recent studies have suggested that taxane chemotherapy may also independently increase the risk of breast cancer related lymphedema
[4-9]. This is important since this class of chemotherapeutic agents is routinely used to treat patients with breast cancer. For example, several studies have shown that patients who undergo taxane chemotherapy have an increased risk of developing lymphedema as compared with patients who were treated with other types of chemotherapy agents (e.g., anthracyclines) or no chemotherapy [4,7-9]. More importantly, a large study of over 5000 breast cancer patients showed that taxane chemotherapy was an independent predictor of arm swelling and symptoms of lymphedema [6]. Although the clinical association between taxane therapy and lymphedema development is strong, there is a paucity of studies that analyze the cellular mechanisms that may account for this association. The goal of this study was therefore to assess the direct effects of docetaxel chemotherapy on lymphatic endothelial cells (LEC). We show that exposure of LECs to varying doses of docetaxel in vitro results in impaired proliferation, tubule formation, and lymphatic migration. In addition, we show that docetaxel exposure decreases expression of lymphatic genes by LECs. Taken together, our results suggest that docetaxel may be toxic to LECs and that even low doses may impair lymphatic function.

${ }^{\star}$ Correspondence to: Babak J Mehrara, MD, Chief, Plastic and Reconstructive Surgery Service, Department of Surgery, Memorial Sloan-Kettering Cancer Center, E-mail: mehrarab@mskcc.org

Key words: docetaxel, lymphatic endothelial cells, chemotherapy, lymphatic markers, LEC death, LEC migration

Received: June 24, 2020; Accepted: July 10, 2020; Published: July 13, 2020 


\section{Materials and methods}

\section{Cell culture}

Primary human dermal LECs (PromoCell, C-12217, Heidelberg, Germany) were grown in Endothelial Cell Growth Medium with penicillin and streptomycin and supplement mix (all from PromoCell) at $37^{\circ} \mathrm{C}$ and $5 \% \mathrm{CO} 2$.

\section{Cell viability assays}

The MTT assay (Thermo Fisher Scientific, V13154, Waltham, MA) quantifies cell viability colorometrically and relies on the fact that the tetrazolium dye MTT 3-(4,5-dimethylthiazol-2-yl)-2,5diphenyltetrazolium bromide is converted by enzymes from live cells to a formazan. LECs $\left(4 \times 10^{4}\right)$ in $200 \mu$ of media were seeded onto 96 well plates overnight. They were then exposed to docetaxel (SelleckChemicals, S1148, Housto n, TX) at varying concentrations $(0,0.01,0.1,1,10,100 \mu \mathrm{M})$ in DMSO for 24 hours at $37^{\circ} \mathrm{C}$. The peak serum concentration $(\mathrm{Cmax})$ of docetaxel in clinical studies is approximately $3 \mu \mathrm{M}[10]$. Because many clinical variables may affect the pharmacokinetics of docetaxel in patients, a wide dose range on a log scale was selected $[11,12]$. The MTT assay was performed according to the manufacturer's recommendation and optical density of the supernatant was measured at $570 \mathrm{~nm}$ by spectrophotometry.

\section{Cell proliferation}

Cell proliferation was assessed using Ki-67 staining. Briefly, LECs $\left(4 \times 10^{4}\right)$ in $400 \mu \mathrm{l}$ of media were seeded onto 4 -well glass chamber slides (Thermo Fisher Scientific, LabTek II) overnight and then treated with docetaxel at varying concentrations $(0,1,10,100 \mu \mathrm{M})$ for 24 hours. The cells were then fixed with $4 \%$ paraformaldehyde (Thermo Scientific, J19943K2) for 15 minutes at room temperature and washed twice with PBS with $0.03 \%$ Tween 20 (PBST; Fisher Scientific, BP337, Hampton, NH). Cells were blocked with $0.03 \%$ PBST with 5\% donkey serum (Southern Biotech, Birmingham, AL) for one hour at room temperature. Rabbit anti-Ki-67 antibody (Abcam, ab16667, Cambridge, MA) was added to LEC and incubated for 5 hours. LECs were washed three times with PBST and stained with Alexa Fluor 594 conjugated donkey anti-rabbit IgG $(\mathrm{H}+\mathrm{L})$ antibody (Thermo Scientific, A-21207) for two hours in the dark. After washing twice with PBST, LECs were exposed for 10 minutes to $0.1 \%$ DAPI for blue nuclear staining, washed, and mounted with a cover slip. Slides were imaged with confocal microscopy (Zeiss, LSM 880, Oberkochen, Germany) and positively stained cells were counted manually. A minimum of 4 wells was analyzed for each dose.

\section{LEC migration}

LEC will migrate to close gaps in a confluent cell culture monolayer. LECs $\left(1 \times 10^{4}\right)$ in $100 \mu \mathrm{l}$ media were seeded for 24 hours on a migration $35 \mathrm{~mm}$ dish with silicone inserts (Ibidi, 81176, Martinsried, Germany) that create a gap in a monolayer of cells. The silicone insert was lifted after 24 hours and the cells were exposed to docetaxel at varying concentrations $(0,1,10,100 \mu \mathrm{M})$. Cells were examined 24 hours of incubation, and the cell migration into the gaps created by the removed inserts was assessed by light microscopy. Light microscopy images were taken of representative samples.

\section{LEC matrigel tube formation assay}

LEC spontaneously form circular, tube-like structures when seeded onto a gel matrix layer, reflecting their ability to collectively organize. Cold liquid Matrigel (Corning, Corning, NY) was used to coat the bottom of a 4-well chamber slide (Lab-Tek II), or a 96 well plate, and allowed to solidify over 30-60 minutes. Docetaxel was added to the LEC $\left(4 \times 10^{4}\right)$ at varying concentrations $(0,1,10$, $100 \mu \mathrm{M})$. LEC were imaged after 3 hours of incubation. Microscopy images were recorded from preset locations on the chamber slide or plate. Individual circular, tube-like, structures were counted manually from fourteen different high-powered fields for each condition.

\section{LEC gene expression}

LECs $\left(3 \times 10^{5}\right)$ were seeded onto 6 -well plates overnight. The cells were washed and then incubated in media containing docetaxel $(0,10,100 \mu \mathrm{M})$ and incubated at $37^{\circ} \mathrm{C}$ for 6 hours. LEC were washed with PBS, lysed with TRizol (Invitrogen) and frozen. Cells were later thawed, vortexed with chloroform, and centrifuged to extract nucleic acids, which were then transferred to $1.5 \mathrm{ml}$ tubes with isopropanol, incubated for 10 minutes and centrifuged. Supernatant was discarded and replaced with $75 \%$ ethanol to precipitate the nucleic acids which were then vortexed, centrifuged, air dried, and resuspended in distilled water. To make cDNA, $8 \mu \mathrm{l}$ RNA, $1 \mu \mathrm{l}$ dsDNase buffer, and $1 \mu \mathrm{l}$ dsDNase (Thermo Fisher Scientific, Waltham, MA) were added to PCR tubes over ice and incubated at $37^{\circ} \mathrm{C}$ for 2 minutes. $4 \mu$ l Maxima cDNA H Minus Synthesis Master Mix (Thermo Fisher Scientific) and $6 \mu$ nucleasefree water were added for reverse transcription. Resulting cDNA was frozen and stored and analyzed for lymphatic cell gene expression by measuring PROX-1, PDPN, LYVE-1 and FLT4.

For quantitative PCR, $1 \mu \mathrm{l} \mathrm{cDNA}$, and $1.25 \mu \mathrm{l}$ primer LYVE1, PDPN, FLT4, or PROX-1 both from Qiagen, Hilden, German) $6.25 \mu \mathrm{l}$ SYBRgreen (Qiagen), and $4.5 \mu \mathrm{l}$ distilled water were added to a 384 well plate for each sample. Primers for glyceraldehyde-3phosphate dehydrogenase (GAPDH) were also used for qPCR of this housekeeping gene as a reference. Samples were run in triplicate, and data was analyzed using Applied Biosystems QuantStudio (Thermo Fisher Scientific).

\section{Statistical analysis}

Statistical analysis was performed using GraphPad Prism (GraphPad Software, Inc., San Diego, CA) software. The results were summarized and presented graphically with means +standard deviation. Analysis between multiple groups was performed using a one-way analysis of variance (ANOVA) with $\mathrm{p}<0.05$ considered significant. Post hoc tests (Dunnett's multiple comparison test) was used to compared treatment and control groups.

\section{Results}

\section{High dose docetaxel is cytotoxic to LECs and decreases cellular proliferation}

Culturing LECs in a high dose of docetaxel $(100 \mu \mathrm{M})$ for 24 hours resulted in significant decrease in cell viability with cell death in more than $80 \%$ of the treated cells as assessed using a MTT assay (Figure 1) $(p<0.01)$. In contrast, exposure to lower doses $(0.01-10 \mu \mathrm{M})$ had minimal effects on cell viability as compared with control LECs.

We next analyzed the expression of Ki67, a marker of cellular proliferation, to determine if treatment with a range of docetaxel doses for 24 hours modulates LEC proliferation (Figure 2). While low doses of docetaxel $(1$ and $10 \mu \mathrm{M})$ did not affect the expression of Ki-67 in LECs, the high dose of doectaxel $(100 \mu \mathrm{M})$ led to a $35 \%$ decline in $\mathrm{Ki}-67$ expression as compared with controls $(\mathrm{p}<0.05)$. 


\section{Docetaxel impairs LEC migration}

Cell migration is the directional motion of a cell in response to a stimulus. LEC are required to migrate during lymphangiogenesis, development, injury response, wound repair, and in response to cancer progression. Since docetaxel has been reported to stabilizes microtubules, preventing their depolymerization and can, as a result, modulate cell motility [10,14], we therefore analyzed LEC migration after exposure to docetaxel by seeding cells with a silicone insert, and then lifting the insert to create a uniform, standardized space between the edges of the LECs in monolayer. By 24 hours, the gaps created by the insert were filled by the untreated LEC that migrated to close the space (Figure 3). In contrast, the gaps persisted for the LEC treated with any dose of docetaxel, even as low as $1 \mu \mathrm{M}$. There did not appear to be any partial responses, and migration appeared to be fully inhibited at all of the doses tested. These results indicate that even low, non-lethal, doses of docetaxel significantly inhibit LEC migration and may therefore impair lymphatic function without causing cellular death.

\section{Docetaxel impairs LEC tubule formation}

Next to determine the effect of docetaxel on the ability of LECs to form connections to nearby cells, we performed a matrigel tubule formation assay. Untreated control LECs form complete, tube-like, ring structures. Every tube is fully intact circle of LECs. Exposure of LECs to low dose docetaxel $(1 \mu \mathrm{M})$ had little effect on tubule formation. However, exposure of cells to an intermediate dose $(10 \mu \mathrm{M})$ resulted

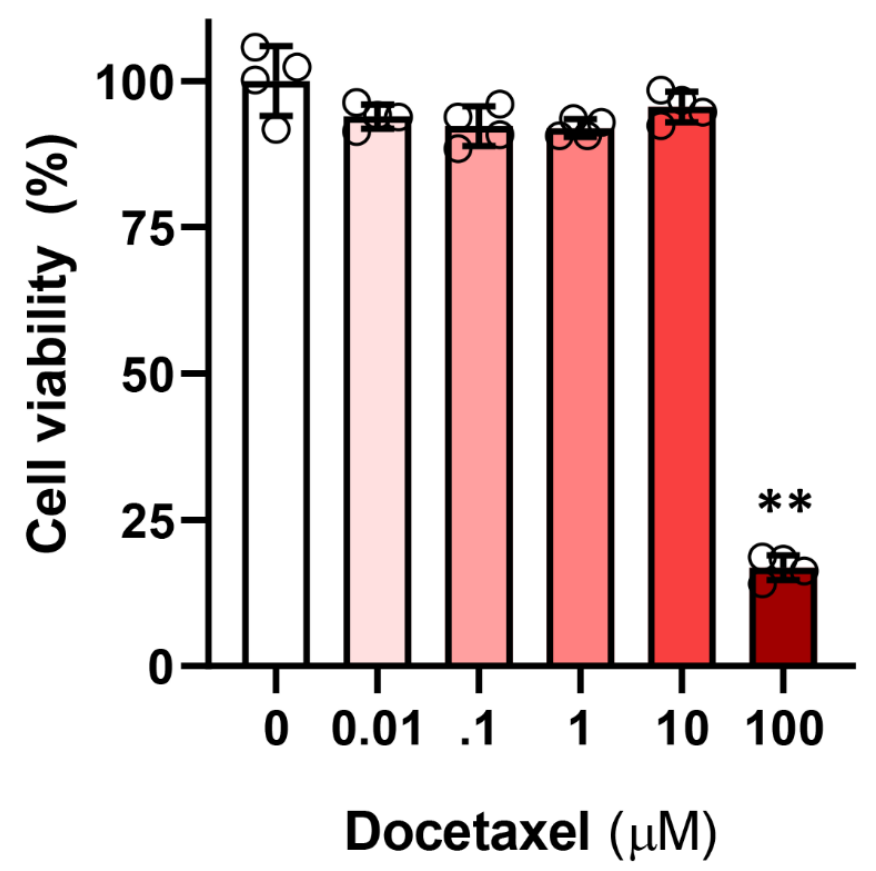

Figure 1. Docetaxel cytotoxicity in LECs. LEC were exposed to varying doses of docetaxel for 24 hours, and then underwent MTT assay to assess cell viability. Control LECs treated with DMSO vehicle containing no decetaxel were considered $100 \%$ viable. $(* * \mathrm{p}<0.01)$

A

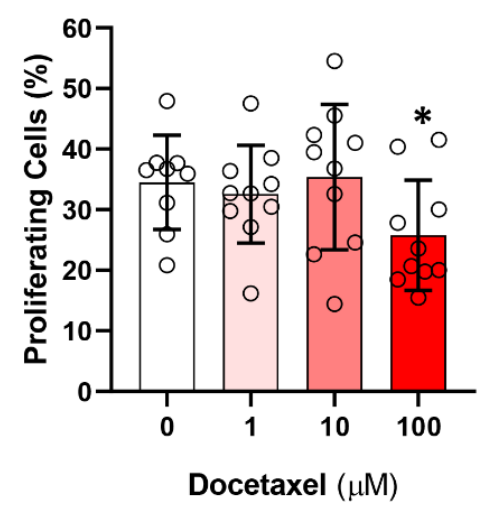

B
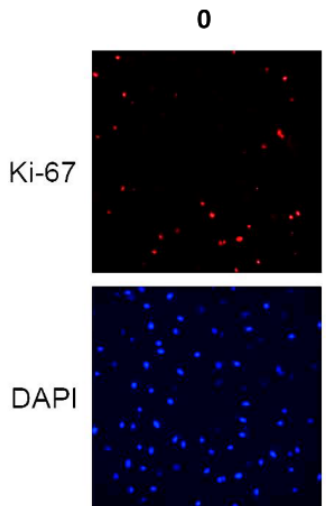

Docetaxel $(\mu \mathrm{M})$

1
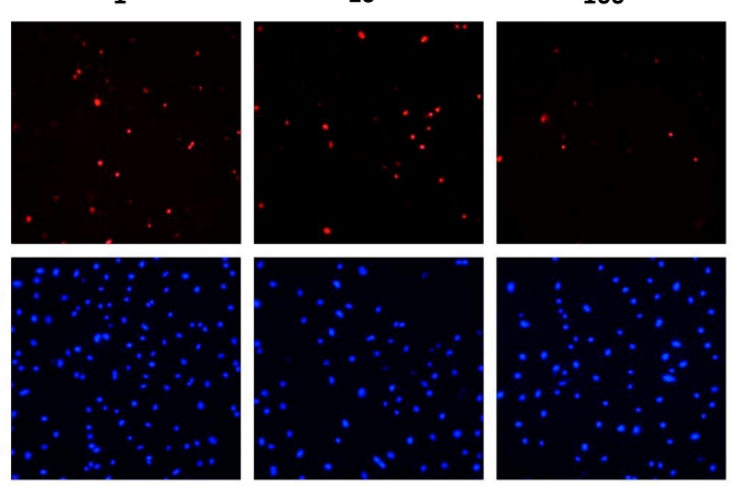

Figure 2. Docetaxel and LEC proliferation. A. LEC were exposed to varying doses of docetaxel for 24 hours and then underwent immunofluorescence staining for Ki-67 to visualize proliferating cells, and DAPI to show all cell nuclei. The proportion of proliferating cells for each group was quantified $\left({ }^{*} \mathrm{p}<0.05\right) \mathbf{B}$. Representative Ki-67 and DAPI immunofluorescence microscopy images are shown 
in significant impairment of tubule formation decreasing the number of intact circular structures by $35 \%(\mathrm{p}<0.05)$ (Figure 4$)$. Treatment with high dose $(100 \mu \mathrm{M})$ docetaxel resulted in a nearly complete loss of tubule formation $(95 \%$ decrease; $(p<0.05))$. LECs treated in this manner failed to organize and took on a rounded appearance consistent with cellular injury and impending death.

\section{Docetaxel impairs LEC gene expression}

Finally, to examine the effect of docetaxel on the expression of lymphatic genes, the mRNA expression of PROX-1, podoplanin (PDPN), LYVE-1, and FLT4 in LECs after 6 hour treatment with docetaxel were analyzed. Treatment with a low dose of docetaxel ( 1 and $10 \mu \mathrm{M})$ had only a modest and insignificant effect on the expression of PROX-1, PDPN, LYVE-1, and FLT4 mRNA. In contrast, high dose docetaxel treatment $(100 \mu \mathrm{M})$ significantly decreased the mRNA expression of PDPN, LYVE-1, and FLT4 but not PROX-1 (Figure 5).

\section{Discussion}

Many recent studies have linked the use of taxane chemotherapy for breast cancer patients with the development of upper extremity lymphedema [4-9]. Although the statistical correlations in these studies are significant, the mechanisms that regulate this potential association remain unknown. In this study we show that high doses of docetaxel are toxic to LECs resulting in cell death and impaired proliferation and expression of lymphatic genes such as PDPN, LYVE-1 and FLT4. More importantly, we found that low or moderate doses of docetaxel, even when applied for a short period of time, have significant effects on LEC migration, and tubule formation.

The toxicity of docetaxel on LECs is likely related to its mechanism of activity on microtubule function. Docetaxel stabilizes microtubules and blocks their ability to depolymerize, which halts their dynamic activity and blocks mitosis. In addition, microtubules are important
A

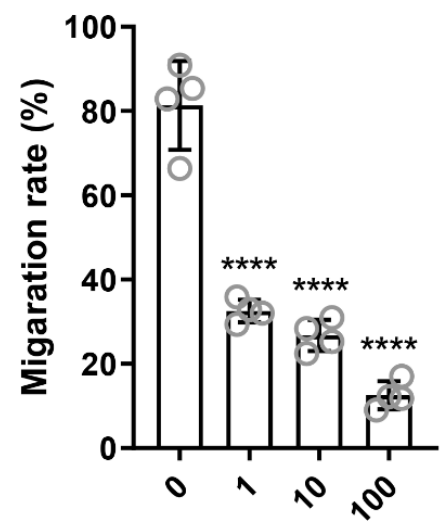

B

Docetaxel $(\mu \mathrm{M})$

0

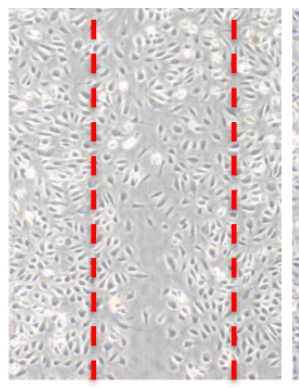

10

$\begin{array}{ccc}1 & 1 \\ 1 & 1 & 1 \\ 1 & 1 \\ 1 & 1\end{array}$

100

$\begin{array}{lll}1 & 1 \\ 1 & 1 \\ 1 & 1 \\ 1 & 1\end{array}$

Docetaxel $(\mu \mathrm{M})$

Figure 3. Docetaxel and LEC migration. A. LEC were seeded as a monolayer and a gap created by a silicone insert that is lifted. LEC were exposed to docetaxel at varying doses for 24 hours. The percentage of cell migration was measured by the ability of the LEC to close the gap $(* * * * p<0.001)$. B. Representative microscopic images are shown. The edges of the cell layer are marked with a dotted red line

A

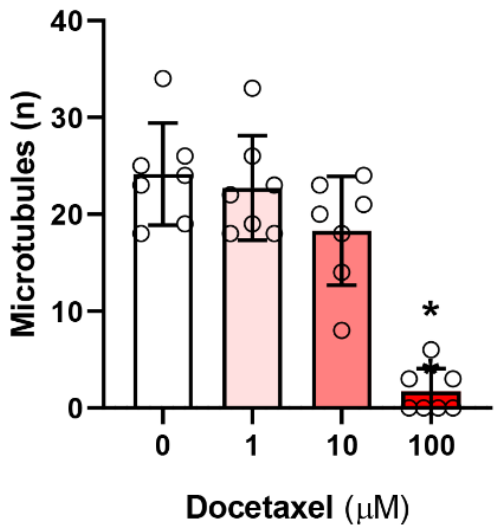

B

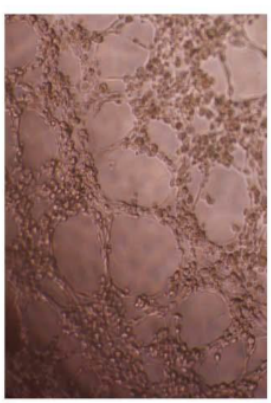

0

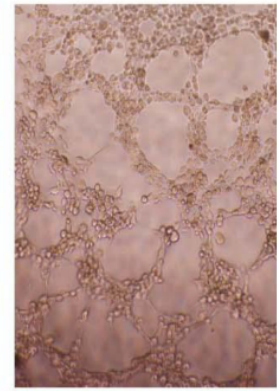

1

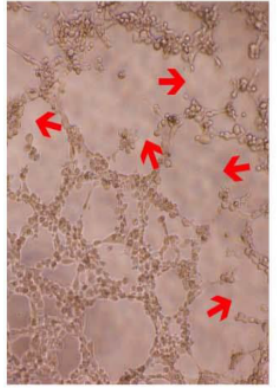

10

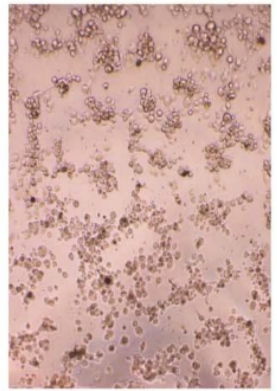

100

Figure 4. Docetaxel and LEC tube formation. A. LEC seeded onto matrigel and incubated in the present of various dose of docetaxel for 3 hours. The number of tubule formation per highpower field image was calculated $(* * \mathrm{p}<0.01)$. B. Representative microscopic images are shown. Red arrow indicates partial tube disruption 

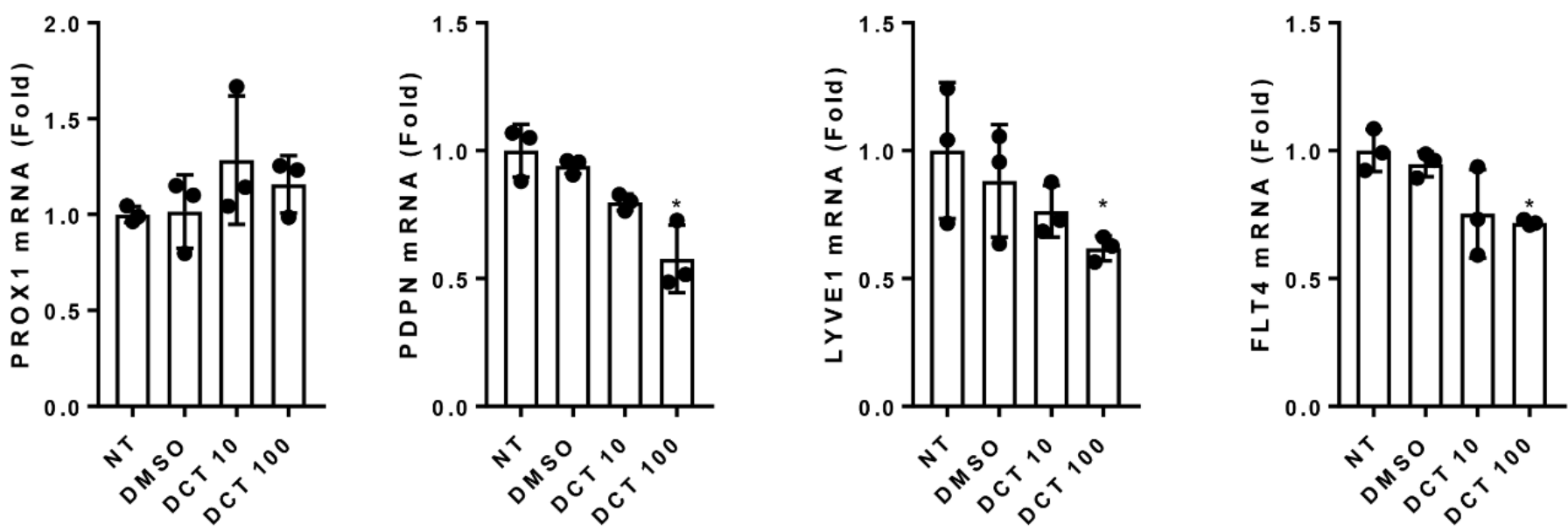

Figure 5. Docetaxel and LEC Gene Expression. LEC were exposed to varying doses of docetaxel for 6 hours and then the mRNA expression of PROX-1, PDPN, LYVE-1, and FLT4 were determined using quantitative real-time PCR $\left({ }^{*} \mathrm{p}<0.05\right)$

contributors to cell migration. Microtubule activity may also directly affect gene expression, and depolymerization of microtubules has been linked to the expression of transcription factors [15].

Importantly, our study suggests that docetaxel induces very different effects on LECs at different doses. The dose required for docetaxel to impair migratory function is the least, followed by the dose necessary to block organization into tubes followed last by the dose necessary for lymphatic gene expression such as FLT4, inducing cell death and blocking proliferation. The implication of these findings is that treatment of breast cancer patient's docetaxel may have different effects on LECs at different doses. Higher serum levels of docetaxel experienced at or around the time of chemotherapy infusion may cause lymphatic injury. In contrast, lower serum levels during troughs of administration may impair lymphatic function. The adverse effects of docetaxel on LEC migration may be the most important determinant of the association between this drug and development of lymphedema since formation of collateral lymphatics following lymphatic occurs normally following axillary lymph node dissection. For example, using a canine model of axillary lymph node dissection, Suami and colleagues showed that collateral lymphatics that bypass the axilla develop shortly after surgery and providing new pathways by which interstitial fluid in the extremity is drained [16]. Thus, even minor alterations in LEC function resulting from taxane chemotherapy may impair collateral lymphatic formation and increase the risk of subsequent lymphedema development. Other studies have also suggested that docetaxel inducedlymphatic dysfunction can modulate the risk of cancer recurrence or metastasis. This hypothesis was based on the finding that docetaxel treatment in a mouse model increased tumor cell survival and invasion by modulating LEC function via complex VEGFR3 dependent and VEGFR3 independent mechansms [17]. Other studies have shown that paclitaxel-another widely used taxane drug- inhibits LEC migration and branch point formation and increased tumor metastasis by inducing autophagy mechanism [18]. Clearly further studies are needed to study these effects; however, our results are intriguing and provide a mechanistic explanation for epidemiological studies demonstrating increased rates of lymphedema in patients treated with taxane chemotherapy.

This study has several limitations. First, the range of docetaxel doses and treatment time that we used in cell culture may not necessarily reflect doses that LECs are exposed to in patients. In addition, docetaxel is typically given weeks before axillary nodal dissection surgery, with repetitive dosing, but its duration of activity in LEC remains poorly understood. A prior clinical study demonstrated that the peak serum concentration (Cmax) is approximately $3 \mu \mathrm{M}$ following administration of $100 \mathrm{mg} / \mathrm{m}^{2}$ intravenously [10]. A review of multiple studies showed that the Cmax ranges between $1.6 \mu \mathrm{g} / \mathrm{ml}$ to $4.1 \mu \mathrm{g} / \mathrm{ml}$ [12], which translates to approximately $1.3 \mu \mathrm{M}$ to $3.3 \mu \mathrm{M}$. The doses used in this study therefore were selected to encompass both above and below this range. Although it should be noted that in patients, the actual amount of docetaxel that reaches the LEC is unclear, it might be possible to be increased in local lymphatic system depending on its delivery methods. Teher Nassar et al. has shown that docetaxel incorporated in PLGA [poly(latic-co-glycolic acid)] nanocapsules is preferentially uptake by lymphatic system and accumulated in mesenteric lymph node from 1.5 hour after its oral administration and continues to exist for 8 hours [19]. More recently, it also has been reported that topical docetaxel treatment leads to higher lymphatic accumulation compared to traditional i.v. delivery method [20]. Moreover, the concertation of docetaxel may depend on many different factors including liver enzyme function, patient age, patient ethnicity, drug-drug interactions, and others $[11,13]$. Finally, it is possible that docetaxel also has significant effects on lymphatic smooth muscle cells that may independently alter the physiology of the lymphatic system and increase the risk of lymphedema.

\section{Conclusion}

In conclusion, we show that docetaxel therapy, even in low doses, has significant effects on LEC migration and function. Higher doses of this drug are cytotoxic to LECs and induce impaired proliferation. These findings provide a possible mechanistic explanation for observations of increased lymphedema in breast cancer patients treated with taxane chemotherapy. Future studies will be required to analyze the effects of taxane therapy in vivo.

\section{Authorship contributions}

BJM and RPK conceived the idea and designed the experiments. AMW and JEB performed all the experiments. AMW, JEB and RPK wrote the manuscript. AMW, JEB, HJP, JS and CLL helped with cell culture, data analysis and manuscript proof reading. 


\section{Competing interests}

Authors declare no conflict of interests.

\section{References}

1. Dayan JH, Ly CL, Kataru RP, Mehrara BJ (2018) Lymphedema: Pathogenesis and novel thrapies. Annu Rev Med 69: 263-276.

2. Tandra P, allam A, Krishnamurthy J (2019) Identification and management of lymphedema in patients with breast cancer. J Oncol Pract 15: 255-262.

3. Rockson SG (2018) Lymphedema after breast cancer treatment. $N$ Engl J Med 379:1 937-1944.

4. Swaroop MN, Ferguson CM, Horick NK, Skolny MN, Miller CL, et al. (2015) Impact of adjuvant taxane-based chemotherapy on development of breast cancer-related lymphedema: results from a large prospective cohort. Breast Cancer Res Treat 151: 393-403.

5. Kim M, Kim SW, Lee SU, Lee NK, Jung SY, et al. (2013) A model to estimate the risk of breast cancer-related lymphedema: combinations of treatment-related factors of the number of dissected axillary nodes, adjuvant chemotherapy, and radiation therapy. Int J Radiat Oncol Biol Phys 86: 498-503.

6. Byun HK, Chang JS, Im SH, Kirova YM, Arsene-Henry A, et al. (2019) Risk of lymphedema following contemporary treatment for breast cancer: An analysis of 7617 consecutive patients from a multidisciplinary perspective. Ann Surg.

7. Jung SY, Shin KH, Kim M, Chung SH, Lee S, et al. (2014) Treatment factors affecting breast cancer-related lymphedema after systemic chemotherapy and radiotherapy in stage II/III breast cancer patients. Breast Cancer Res Treat 148: 91-98.

8. Zhu W, Li D, Li X, Ren J, Chen W, et al. (2017) Association between adjuvant docetaxel- based chemotherapy and breast cancer-related lymphedema. Anticancer Drugs 28: 350-355.

9. Lee MJ, Beith J, Ward L, Kilbreath S (2014) Lymphedema following taxane-based chemotherapy in women with early breast cancer. Lymphat Res Biol 12: 282-288.
10. De Weger VA, Beijnen JH, Schellens JH (2014) Cellular and clinical pharmacology of the taxanes docetaxel and paclitaxel--a review. Anticancer Drugs 25: 488-494.

11. Nieuweboer AJ, de Morree ES, de Graan AJ, Sparreboom A, de Wit R, et al. (2015) Inter-patient variability in docetaxel pharmacokinetics: A review. Cancer Treat Rev 41 605-613.

12. Kenmotsu H, Tanigawara Y (2015) Pharmacokinetics, dynamics and toxicity of docetaxel: Why the Japanese dose differs from the Western dose. Cancer Sci 106: 497504.

13. Schonmeyr BH, Wong AK, Soares M, Fernandez J, Clavin N, et al. (2008) Ionizing radiation of mesenchymal stem cells results in diminution of the precursor pool and limits potential for multilineage differentiation. Plast Reconstr Surg 122: 64-76.

14. Hernandez-Vargas H, Palacios J, Moreno-Bueno G (2007) Telling cells how to die: docetaxel therapy in cancer cell lines. Cell Cycle 6: 780-783.

15. Rosette C, Karin M (1995) Cytoskeletal control of gene expression: depolymerization of microtubules activates NF-kappa B. J Cell Biol 128: 1111-1119.

16. Suami H, Yamashita S, Soto-Miranda MA, Chang DW (2013) Lymphatic territories (lymphosomes) in a canine: an animal model for investigation of postoperative lymphatic alterations. PLoS One 8: e69222.

17. Harris AR, Perez MJ, Munson JM (2018) Docetaxel facilitates lymphatic-tumor crosstalk to promote lymphangiogenesis and cancer progression. BMC Cancer 18: 718 .

18. Zamora A, Alves M, Chollet C, Therville N, Fougeray T, et al. (2019) Paclitaxel induces lymphatic endothelial cells autophagy to promote metastasis. Cell Death Dis 10: 956.

19. Nassar T, Attili-Qadri S, Harush-Frenkel O, Farber S, Lecht S, et al. (2011) High plasma levels and effective lymphatic uptake of docetaxel in an orally available nanotransporter formulation. Cancer Res 71: 3018-3028.

20. Worley DR, Hansen RJ, Wittenburg LA, Chubb LS, Gustafson DL, et al. (2016) docetaxel accumulates in lymphatic circulation following subcutaneous delivery compared to intravenous delivery in rats. Anticancer Res 36: 5071-5078.

Copyright: $\odot 2020$ Wong AM. This is an open-access article distributed under the terms of the Creative Commons Attribution License, which permits unrestricted use, distribution, and reproduction in any medium, provided the original author and source are credited. 